\title{
Targeted Radiotherapeutics from 'Bench-to-Bedside'
}

\author{
Cristina Müllera, Martin Béhéa, Susanne Geistlicha, Nicholas P. van der Meulen ${ }^{\text {ab }}$, and \\ Roger Schibli ${ }^{\star a c}$
}

\begin{abstract}
The concept of targeted radionuclide therapy (TRT) is the accurate and efficient delivery of radiation to disseminated cancer lesions while minimizing damage to healthy tissue and organs. Critical aspects for successful development of novel radiopharmaceuticals for TRT are: i) the identification and characterization of suitable targets expressed on cancer cells; ii) the selection of chemical or biological molecules which exhibit high affinity and selectivity for the cancer cell-associated target; iii) the selection of a radionuclide with decay properties that suit the properties of the targeting molecule and the clinical purpose. The Center for Radiopharmaceutical Sciences (CRS) at the Paul Scherrer Institute in Switzerland is privileged to be situated close to unique infrastructure for radionuclide production (high energy accelerators and a neutron source) and access to C/B-type laboratories including preclinical, nuclear imaging equipment and Swissmedic-certified laboratories for the preparation of drug samples for human use. These favorable circumstances allow production of non-standard radionuclides, exploring their biochemical and pharmacological features and effects for tumor therapy and diagnosis, while investigating and characterizing new targeting structures and optimizing these aspects for translational research on radiopharmaceuticals. In close collaboration with various clinical partners in Switzerland, the most promising candidates are translated to clinics for 'first-in-human' studies. This article gives an overview of the research activities at CRS in the field of TRT by the presentation of a few selected projects.
\end{abstract}

Keywords: Folate, PSMA · Minigastrin · Radiopharmacy · Targeted radionuclide therapy · Terbium radionuclides $\cdot$ Theragnostics

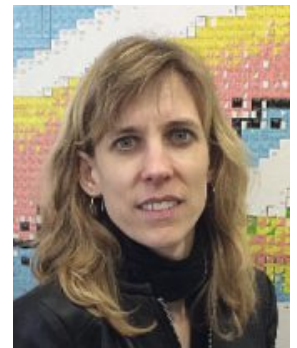

PD Dr. Cristina Müller is a research group leader at the Center for Radiopharmaceutical Sciences at Paul Scherrer Institute. She studied Pharmaceutical Sciences at the University of Bern and ETH Zurich. She has been focusing her research activities on the development and preclinical application of novel radioligands for targeting cancer and inflammatory diseases. Her particular interest lies in the investigation of 'exotic' radionuclides for the optimization of the radiotheragnostic concept.

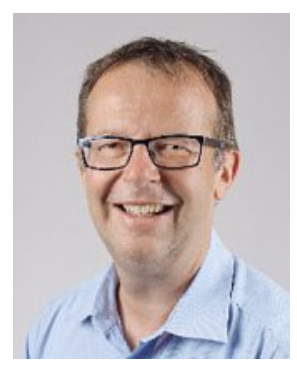

Dr. Martin Béhé received his PhD in inorganic chemistry at the University of Basel. He worked at the University Hospitals of Göttingen, Marburg and Freiburg in Germany where he established a research group for peptide-based radiopharmaceuticals and its production. Since 2011, he leads the Pharmacology group at the Center for Radiopharmaceutical Sciences at PSI, where the Araris Linker Technology took its first steps. He has an extensive background in peptide chemistry, peptide labeling and conjugation as well and critically contributed to development of radio-labeled compounds that are used in the clinic.

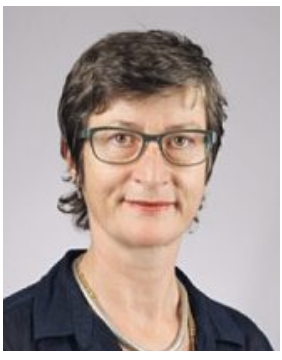

Susanne Geistlich graduated as Pharmacist from ETH Zurich and joined the Center for Radiopharmaceutical Sciences at PSI and ETH in 2009 after having gained experience in the pharmaceutical industry. She obtained the EANM certificate as a radiochemist/radiopharmacist in 2011. She heads the group responsible for translating radiopharmaceuticals developed at CRS from preclinical to clinical use and her team supplies clinical trials with study drugs.

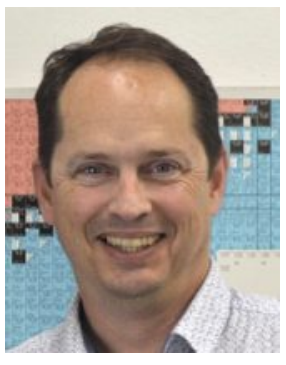

Dr. Nicholas P. van der Meulen studied chemistry at Stellenbosch University, South Africa, obtaining his PhD in 2008. He worked at iThemba LABS, South Africa, as a research chemist in the Radionuclide Production group for 17 years before accepting the position of Group Leader at PSI in 2013. Currently he leads the Radionuclide Development group as a shared endeavor between the Laboratory of Radiochemistry and the Center for Radiopharmaceutical Sciences. The main research focus lies in the development of 'exotic' radionuclides in view of a clinical application.

\footnotetext{
${ }^{*}$ Correspondence: Prof. R. Schibliac, E-mail: roger.schibli@psi.ch

aCenter for Radiopharmaceutical Sciences, Forschungsstrasse 111, Paul Scherrer Institute, CH-5232 Villigen-PSI, Switzerland;

'Laboratory of Radiochemistry, Forschungsstrasse 111, Paul Scherrer Institute, CH-5232 Villigen-PSI, Switzerland,

'Department of Chemistry and Applied Biosciences, ETH Zurich, Vladimir-Prolog-Weg 1-5/10, CH-8093 Zurich, Switzerland
} 


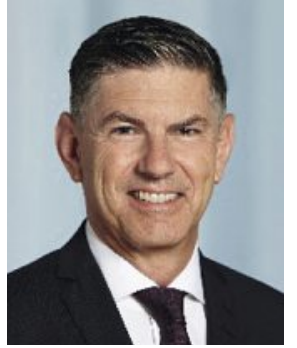

Prof. Dr. Roger Schibli is Full Professor in the Department of Chemistry and Applied Biosciences (ETH Zurich) since 2010, and Laboratory Head at the Paul Scherrer Institute (PSI). He leads the Center for Radiopharmaceutical Science (CRS), a joint endeavor between ETH Zurich, Paul Scherrer Institute and the University Hospital Zurich. Prof. Schibli studied Chemistry at the University of Basel. His research is directed towards targeted tumor diagnosis and therapy using radiolabeled compounds from 'bench-to-bedside'.

\section{Introduction}

Targeted radionuclide therapy (TRT) is a promising therapeutic modality for patients with non-operable tumors and tumor metastases. As compared to 'standard care' chemotherapy and external beam radiotherapy, TRT is thought to have fewer adverse events and improved efficiency through targeting tumor-associated proteins such as receptors, antigens or membrane-associated transporters.

The development of radiolabeled molecules that target such cancer-related structures is a multidisciplinary approach. Expertise in physics, radiochemistry, inorganic and organic chemistry, biochemistry as well as pharmacology, pharmacy and medicine are required. Chemical and biological lead structures with high affinity and selectivity for a clinically relevant target have to be identified. These lead structures are specifically modified to enable incorporation of the corresponding radionuclide without affecting the pharmacokinetic properties and/or target binding affinity of the radiopharmaceutical. The selection of an appropriate radionuclide is also critical, as it may have a profound impact on functionalization strategies (e.g. covalent radiolabeling or via chelating agents suitable for radiometals) of a targeting molecule as well as on the in vitro and in vivo properties of the future radiopharmaceutical. Subsequently, the radiopharmaceuticals are evaluated in vitro and in preclinical animal studies, including non-invasive small animal nuclear imaging. Promising radiopharmaceuticals may qualify for clinical trials once toxicological tests have been performed and robust and reproducible manufacturing processes have been established according to the rules of Good Manufacturing Practice (GMP) (Fig. 1).

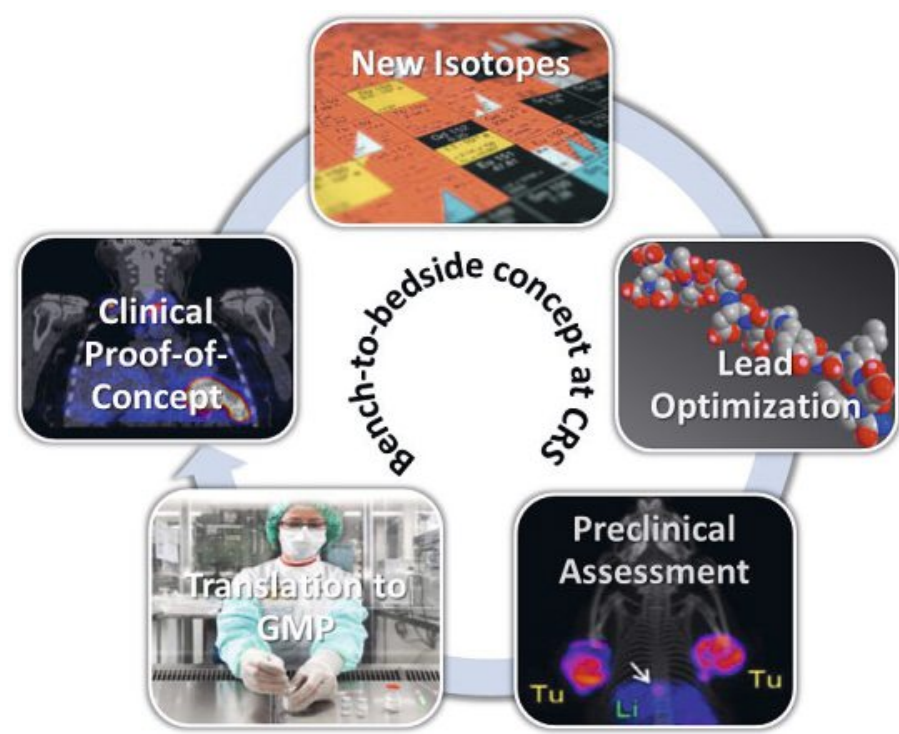

Fig. 1. Translational ('bench-to-bedside'), radiopharmaceutical research includes: i) development of new radionuclides for diagnosis and therapy; ii) lead optimization; iii) in vivo, preclinical evaluation of new radiopharmaceuticals; iv) production of radiopharmaceuticals in clean rooms; v) clinical trials.
The expensive infrastructure (e.g. cyclotron facilities, neutron sources, Type A/B/C laboratories, and Swissmedic-certified laboratories for the preparation of drug samples for human use) and the highly specialized and complex know-how in accelerator technology, accelerator physics, target development, radiochemistry, radiation protection and radiosyntheses suggest that radiopharmaceutical research in Switzerland typically takes place in an academic environment. On the other hand, the sometimes extremely short half-lives of the employed radionuclides favor the physical proximity of the facilities to the clinics. Recently, Big Pharma began showing great interest in radiopharmaceuticals for cancer therapy, in particular. Bayer successfully launched the alpha-particle emitter ${ }^{223} \mathrm{RaCl}_{2}$ (Xofigo ${ }^{\circledR}$ ) for the treatment of osseous metastases in castration-resistant prostate cancer patients (CRPC), ${ }^{[1]}$ while Novartis acquired Advanced Accelerator Applications in 2018 after they completed a successful Phase III clinical trial (NETTER-1 trial) with a [177 Lu]Lu-labeled somatostatin analogue $\left(\left[{ }^{177} \mathrm{Lu}\right] \mathrm{Lu}-\mathrm{DOTATATE}\right.$, Lutathera $\left.^{\circledR}\right)$ for the treatment of metastasized neuroendocrine tumors. ${ }^{[2]}$ In the same year, Novartis acquired Endocyte after they licensed $\left[{ }^{177} \mathrm{Lu}\right] \mathrm{Lu}-$ PSMA-617, a potential first-in-class radiopharmaceutical for the treatment of metastatic castration-resistant prostate cancer patients (mCRPC) targeting the prostate-specific membrane antigen (PSMA). ${ }^{[3]}\left[{ }^{177} \mathrm{Lu}\right] \mathrm{Lu}-\mathrm{PSMA}-617$ is currently being investigated in the Phase III global VISION clinical trial (ClinicalTrials.gov: NCT03511664). [4]

At Paul Scherrer Institute (PSI), the Center for Radiopharmaceutical Sciences (CRS) focuses on the development of novel radiopharmaceuticals targeting the cholecystokinin $\mathrm{B}$ receptor (CCKBR) and the folate receptor (FR), in particular. At the same time, CRS is optimizing the structure of PSMAtargeting agents with the aim to improve their pharmacological profiles and, hence, increase their tumor uptake. Furthermore, CRS has investigated the improved efficacy of the clinically-used PSMA-617 with the engagement of innovative, therapeutic radionuclides. This article describes selected examples of the research endeavors at CRS, some of which were carried out together with other academic research groups as well as industrial partners.

\section{Developing Minigastrin Analogues from 'Bench-to- Bedside'}

In the early $1940 \mathrm{~s}$, radioactive iodide $\left({ }^{131} \mathrm{I}^{-}\right)$was used for treatment of patients with hyperthyroidism as the first systemic radiotherapy. Since 1946, radioactive iodide has become a standard diagnostic and therapeutic radiopharmaceutical for papillary or follicular thyroid cancers, ${ }^{[5]}$ however, not all thyroid cancer types take up radioactive iodide. The most notable example in this regard is medullary thyroid cancer (MTC), which does not accumulate iodide due to its origin in parafollicular C-cells. ${ }^{[5]}$ Limited therapy options are, therefore, available. It is well documented, however, that MTC cells frequently express CCKBR to which gastrin binds with high affinity. ${ }^{[6]}$ Radiolabeled gastrin analogues may, thus, serve as a therapeutic option for these patients. The initial gastrin derivatives suffered from a high kidney retention, which prohibited a therapeutic application due to potential radionephrotoxicity (e.g. DTPAMG0, ${ }^{[7]}$ Table 1). The glutamic acid chain in the linker entity was identified as the reason for the high kidney uptake observed. As part of a European COST Action (BM0607) consortium, twelve radiolabeled CCKBR ligands were tested for their binding affinity, stability and biodistribution. ${ }^{[8]}$ The ${ }^{111} \operatorname{In}[\mathrm{In}]-$ labeled peptides comprising Gln (PPF10) and DGlu 6 (PPF11) linker entities, respectively, showed the best in vivo behavior with respect to high tumor uptake and low kidney retention. This situation resulted in a tumor-to-kidney ratio of 3.1 and 1.5 , respectively, compared to 0.3 determined for the reference peptide ${ }^{111} \mathrm{In}[\mathrm{In}]-\mathrm{MG} 0$. The results showed that the change

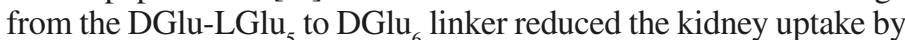
about $90 \%$, with a retained high tumor uptake of $6.44 \% \mathrm{IA} / \mathrm{g}$. 


\begin{tabular}{l|l}
$\begin{array}{l}\text { Peptide } \\
\text { Minigastrin }\end{array}$ & Amino acid sequence \\
\hline MG0 & DOTA-DGlu-Glu-Glu-Glu-Glu-Glu-Ala-Tyr-Gly-Trp-Met-Asp-Phe-NH ${ }_{2}$ \\
\hline DTPAMG0 & DTPA-DGlu-Glu-Glu-Glu-Glu-Glu-Ala-Tyr-Gly-Trp-Met-Asp-Phe-NH \\
\hline PPF10 & DOTA-DGln-DGln-DGln-DGln-DGln-DGln-Ala-Tyr-Gly-Trp-Met-Asp-Phe-NH \\
\hline PPF10N & DOTA-DGln-DGln-DGln-DGln-DGln-DGln-Ala-Tyr-Gly-Trp-Nle-Asp-Phe-NH \\
\hline PPF11 & DOTA-DGlu-DGlu-DGlu-DGlu-DGlu-DGlu-Ala-Tyr-Gly-Trp-Met-Asp-Phe-NH \\
\hline PPF11N & DOTA-DGlu-DGlu-DGlu-DGlu-DGlu-DGlu-Ala-Tyr-Gly-Trp-Nle-Asp-Phe-NH \\
\hline
\end{tabular}

Table 1. Amino acid sequences of native minigastrin and the different gastrin analogs (italic indicates structural changes compared to the lead compound MGO).
The sulfo-ether of the methionine side chain in peptides PPF10 and PPF11 was easily oxidized during the radiolabeling procedure, leading to a significant loss of affinity towards the CCKBR (10-40-fold reduction of $\mathrm{IC}_{50}$ values compared to the non-oxidized radiopeptides). Methionine was, therefore, replaced by a norleucine, resulting in the peptides PPF11N and PPF10N (Table 1). The ${ }^{177} \mathrm{Lu}$-labeled PPF11N and PPF11N were compared with ${ }^{177} \mathrm{Lu}[\mathrm{Lu}]-$ PPF10, ${ }^{177} \mathrm{Lu}[\mathrm{Lu}]-\mathrm{PPF} 11$ as well as with the respective oxidized derivatives for receptor affinity by competition assay and biodistribution studies in mice bearing CCKBR-positive A431 tumors. ${ }^{[9]}$ The biodistribution results of ${ }^{177} \mathrm{Lu}[\mathrm{Lu}]-\mathrm{PPF} 11 \mathrm{~N}$ showed an increased tumor uptake compared to ${ }^{177} \mathrm{Lu}[\mathrm{Lu}]-\mathrm{PPF} 11$ by a factor of more than 2 (from $4.7 \pm 1.8 \% \mathrm{IA} / \mathrm{g}$ to $11.0 \pm 2.5 \% \mathrm{IA} / \mathrm{g}$ ), which was not observed for the ${ }^{177} \mathrm{Lu}[\mathrm{Lu}]-\mathrm{PPF} 10$ derivative (Fig. 2).

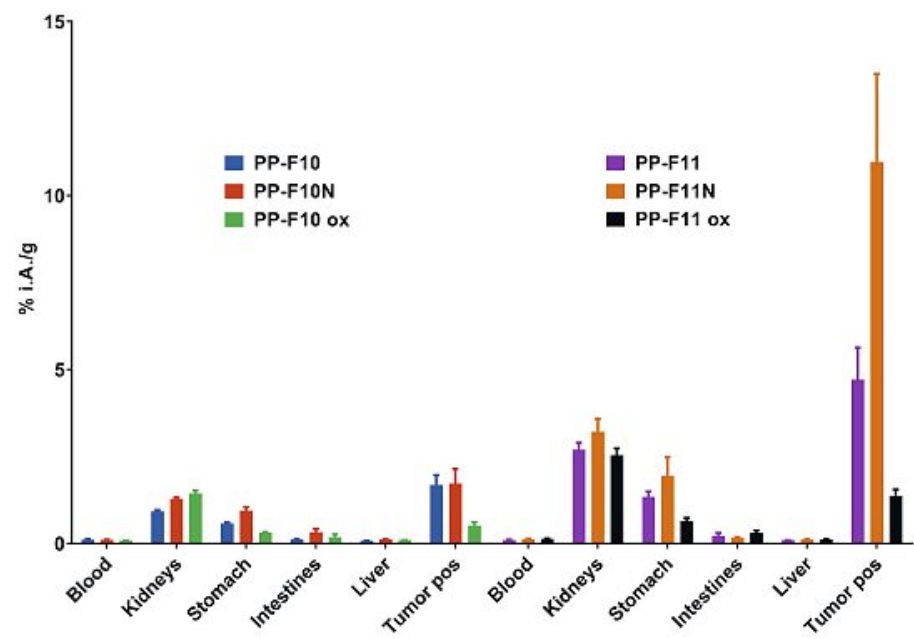

Fig. 2. Biodistribution data of [ $\left.{ }^{177} \mathrm{Lu}\right] \mathrm{Lu}-\mathrm{PPF} 10$ and $\left[{ }^{177} \mathrm{Lu}\right] \mathrm{Lu}-\mathrm{PPF} 11$ and their corresponding Nle-derivatives ( $\left.{ }^{177} \mathrm{Lu}\right] \mathrm{Lu}-\mathrm{PPF} 10 \mathrm{~N}$ and $\left[{ }^{177} \mathrm{Lu}\right] \mathrm{Lu}-$ PPF11N) and those with the oxidized methionine ([177Lu]Lu-PPF10ox, [177Lu]Lu-PPF10ox) in nude mice bearing CCKBR-transfected A431 xenografts. The oxidized version lost affinity to CCKBR, which is reflected by a low tumor uptake. There was no change observed in the tumor uptake between $\left[{ }^{177} \mathrm{Lu}\right] \mathrm{Lu}-\mathrm{PPF} 10$ and $\left[{ }^{177} \mathrm{Lu}\right] \mathrm{Lu}-\mathrm{PPF} 10 \mathrm{~N}$ whereas the change from [ $\left.{ }^{177} \mathrm{Lu}\right] \mathrm{Lu}-\mathrm{PPF} 10$ to $\left[{ }^{177} \mathrm{Lu}\right] \mathrm{Lu}-\mathrm{PPF} 10 \mathrm{~N}$ increased the tumor uptake by more than two times.

A clinical study with patients suffering from metastasized MTC was initiated, in collaboration with the Department of Nuclear Medicine at University Hospital Basel, in 2015 (ClinicalTrials. gov: NCT02088645). The GMP-production of ${ }^{177} \mathrm{Lu}[\mathrm{Lu}]-\mathrm{PPF} 11 \mathrm{~N}$ was performed at PSI and the sterile injection solution sent to the University Hospital Basel for application within $24 \mathrm{~h}$ after production. The patients were initially injected with low activity of radiopeptide for diagnostic and dosimetric purposes. The diagnostic study was promising in different aspects: i) the calculated absorbed dose to the tumor and healthy organs favored therapeutic application and; ii) with the ${ }^{177} \mathrm{Lu}[\mathrm{Lu}]-\mathrm{PPF} 11 \mathrm{~N}$, cancer lesions that were not detected with computer tomography (CT) or magnetic resonance imaging (MRI) were visualized using scintigraphy and single proton emission computer tomography (SPECT; (Fig. 3). ${ }^{[10]}$ A phase I/b dose escalation study is currently ongoing. ${ }^{177} \mathrm{Lu}[\mathrm{Lu}]-\mathrm{PPF} 11 \mathrm{~N}$ was recently licensed to Debiopharm - a pharmaceutical company based in Switzerland.

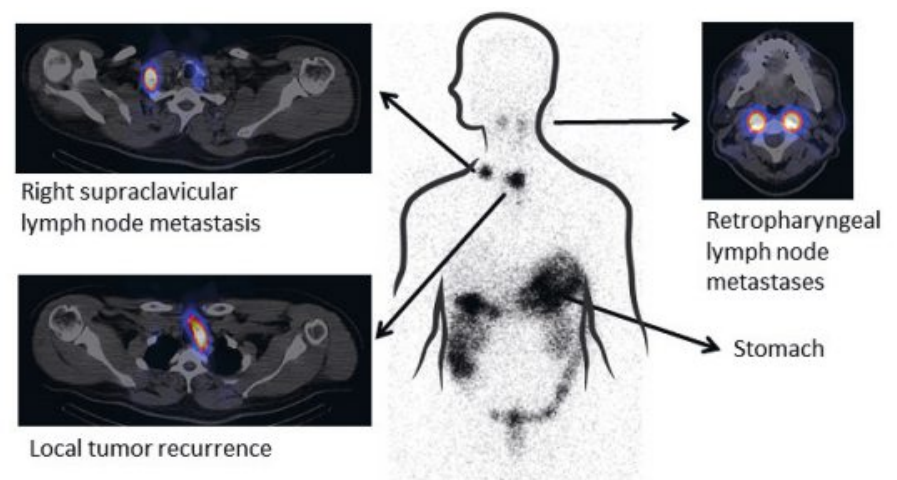

Fig. 3. (Center) Scintigraphy of patient performed $24 \mathrm{~h}$ after injection of $\left[{ }^{177} \mathrm{Lu}\right] \mathrm{Lu}-\mathrm{PPF} 11 \mathrm{~N}$. Specific uptake in the metastases of a medullary thyroid carcinoma and the stomach selected SPECT/CT in transversal slices (Courtesy of the Dept. of Nuclear Medicine at the University Hospital Basel, Prof. D. Wild and Dr. Ch. Rottenburger).

\section{Exploring New Therapeutic Radionuclides at PSI}

Ideally, the type of radionuclide used in a therapeutic radiopharmaceutical is tailored to the specific type of cancer or even the stage of the disease. Radionuclides that undergo $\alpha$-decay produce particles composed of two neutrons and two protons, whereas radionuclides that undergo $\beta^{-}$-decay emit energetic electrons from their nuclei.[11] Some radionuclides can also emit conversion or Auger electrons via secondary effects. $\alpha$-Particles have high linear energy transfer (LET) - between 20-190 keV/ $\mu \mathrm{m}$ - and are capable of damaging DNA both directly and via the production of radicals and reactive oxygen species. $\beta^{-}$-particles have lower LET values of $0.2-2.0 \mathrm{keV} / \mu \mathrm{m}$ and mainly exert their therapeutic action via production of free radicals in the cancer cell. Conversion

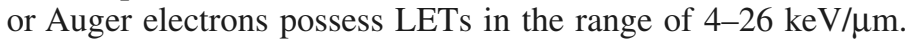
Particles with high and medium LET can be useful for TRT depending on the size of the metastases and on the radiosensitivity of the tumor cells. For example, the TRT of leukemia and lymphoma typically requires particles of lower energy than it would be the case for TRT of solid tumors (Fig. 4).

Therefore, the radionuclides' physical properties significantly contribute to a satisfactory therapeutic outcome in TRT. Today, only a limited number of therapeutic radionuclides are available for routine clinical TRT (e.g. yttrium-90, lutetium-177, iodine-131, radium-223). Recently, researchers have started to 


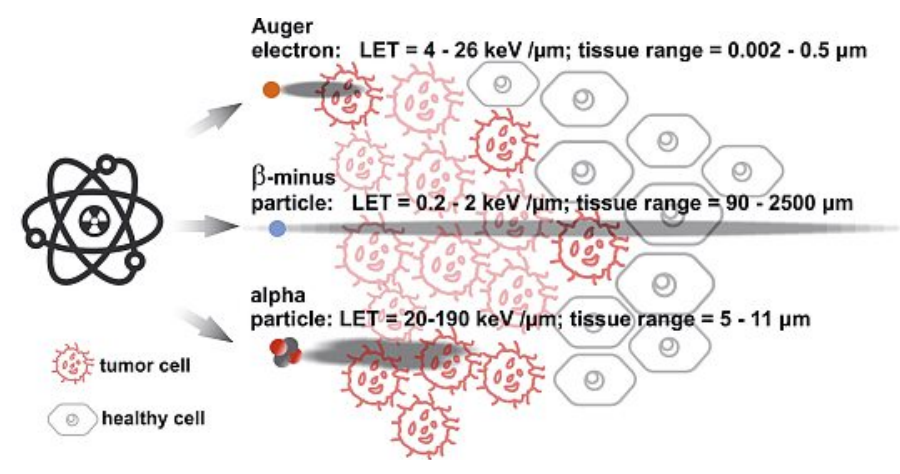

Fig. 4. Schematic presentation of the range of $\alpha-$, $\beta^{-}$-particles and Auger (and conversion) electrons in tissue and their corresponding linear energy transfer (LET) ranges.

explore the realm of suitable radionuclides for potential radiopharmaceutical and nuclear medical applications. In particular, radionuclides with medium and high LET are the focus of attention. The Radionuclide Development group at PSI (a shared group between CRS and Laboratory of Radiochemistry) ${ }^{[12]}$ strives to produce novel radionuclides of high purity with specific decay properties that are superior to those that are currently used in the clinics. The in vitro and in vivo characterization and application of these radionuclides, in combination with different targeting molecules, takes place at CRS. The interest of CRS lies, in particular, in the production and investigation of radionuclides of the same element, so-called radioisotopes, that can be employed either for diagnosis or therapy and, thus, realizing the radiotheragnostics concept using chemically identical radiopharmaceuticals. Among the most interesting elements in this regard are scandium and terbium, as they comprise several interesting radioisotopes for imaging purposes (scandium-43/44 and terbium-152/155) and therapeutic application (scandium-47 and terbium-149/161). ${ }^{[13]}$ Scandium and terbium radionuclides are more suited than the use of pairs of unrelated radiometals, such as gallium-68 and lutetium-177 that are currently used in routine clinical interventions for positron emission tomography (PET) imaging and TRT. Terbium is also of interest to compare effects of different types of particle emission for therapeutic purposes. Terbium-161 emits $\beta^{-}$particles and conversion and Auger electrons, while terbium-149 emits $\alpha$-particles. They may serve for an in-depth investigation of therapeutic effects using chemically identical radiopharmaceuticals to understand the advantages and disadvantages of these therapy concepts. Both of these radionuclides have great potential for clinical translation, as demonstrated in our preclinical studies described in the sections that follow.

\subsection{Terbium-161: A Potentially more Powerful Alternative to Lutetium-177}

A major focus of our research activities at the PSI over the last decade has been the production and investigation of the terbium radioisotopes. ${ }^{[14]}$ Terbium is unique in that it comprises four medically-interesting radioisotopes for all four modalities in nuclear medicine. This includes positron emission tomography (PET, terbium-152) and SPECT (terbium-155) imaging as well as targeted $\alpha$ - (terbium-149) and $\beta^{-}$-radionuclide therapy (terbium-161). At this development stage, terbium-161 is the most advanced radioisotope of the terbium family, not only in terms of production and preclinical investigations, but also in view of a clinical translation. Herein, we summarize and discuss the achievements made with terbium-161 at CRS and in collaboration with our external partners.

Terbium-161 decays with a half-life of $6.953 \mathrm{~d}$ (recently determined by Duran et al. ${ }^{[15]}$ ) by the emission of medium-energy $\beta^{-}$-particles $\left(\mathrm{E} \beta^{-}=154 \mathrm{keV}\right)$ and $\gamma$-radiation $(\mathrm{E} \gamma=48 \mathrm{keV}$, I $=17.0 \%), 75 \mathrm{keV}, \mathrm{I}=10.2 \%)$. In this regard, terbium-161 closely resembles lutetium-177 $\left(\mathrm{T}_{1 / 2}=6.65 \mathrm{~d}, \mathrm{E} \beta^{-}{ }_{\text {average }}=134 \mathrm{keV}, \mathrm{E} \gamma=\right.$ $113 \mathrm{keV}, \mathrm{I}=6.2 \%$ and $208 \mathrm{keV}, \mathrm{I}=10.4 \%$ ), the radiometal currently most often employed for TRT in clinics. ${ }^{[16]}$ The medium energy $\beta^{-}$-particles of lutetium-177 were revealed to be favorable for the treatment of metastases, while preventing radionephrotoxicity, as previously observed when using yttrium-90, a highenergy $\beta^{-}$-particle emitter. ${ }^{[17]}$ Terbium-161 is considered superior to lutetium-177, due to the broader spectrum of emitted electrons. In addition to the $\beta^{-}$-particles, terbium-161 also emits a substantial number of short-range electrons, so-called conversion and Auger electrons. These high-LET particles are of unique value for the killing of single cancer cells, as they deliver a 3- to 4-fold increased dose to spheres of subcellular dimensions..$^{[18]}$

In an initial preclinical study, terbium-161 and lutetium-177 were compared using a DOTA-folate conjugate as a tumor-targeting agent (Fig. 5A). ${ }^{[19]}$ It was successfully demonstrated that $\left[{ }^{161} \mathrm{~Tb}\right] \mathrm{Tb}$-DOTA-folate reduced KB tumor cell viability more effectively than $\left[{ }^{177} \mathrm{Lu}\right] \mathrm{Lu}-\mathrm{DOTA}$-folate when applied at the same activity concentration (Fig. 5B). Even more intriguing was the observation that the tumor growth was more delayed in mice treated
Fig. 5. (A) Chemical structure of the DOTA-folate conjugate (cm09).

(B) Inhibitory effects of increasing activity concentrations of [ $\left.{ }^{161} \mathrm{~Tb}\right]$ Tb-DOTA-folate ( $\mathrm{IC}_{50}$ value: 0.014 $\pm 0.013 \mathrm{MBq} / \mathrm{mL})$ and $\left[{ }^{177} \mathrm{Lu}\right] \mathrm{Lu}-$ DOTA-folate $\left(\mathrm{IC}_{50}\right.$ value: $0.063 \pm$ $0.021 \mathrm{MBq} / \mathrm{mL}$ ) in $\mathrm{KB}$ tumor cells in vitro. (C) Survival curve of mice treated with [ $\left.{ }^{161} \mathrm{~Tb}\right] \mathrm{Tb}$-DOTA-folate (median survival: 54 days) and [ $\left.{ }^{177} \mathrm{Lu}\right]$ Lu-DOTA-folate (median survival: 35 days) as compared to untreated controls (median survival: 31 days); (D) SPECT/CT images shown as maximum intensity projections (MIPs) $30 \mathrm{~h}$ after injection of about $30 \mathrm{MBq}\left[{ }^{161} \mathrm{~Tb}\right] \mathrm{Tb}-\mathrm{DOTA}-$ folate and [ $\left.{ }^{177} \mathrm{Lu}\right]$ Lu-DOTA-folate, respectively. The data were adapted from Müller et al., 2014, Eur J Nucl Med Mol Imaging. ${ }^{[19]}$
A

Structure: DOTA-folate

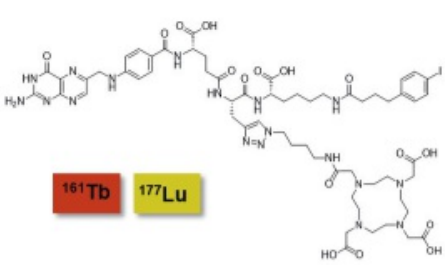

C

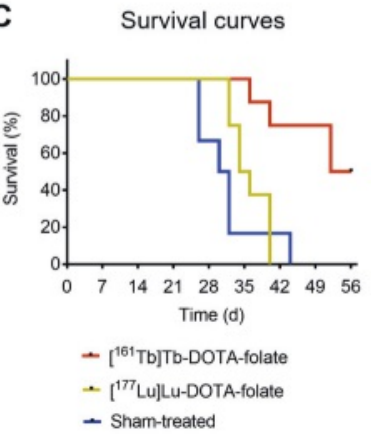

B

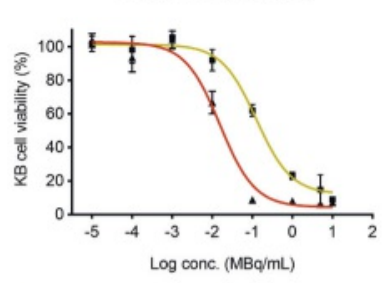

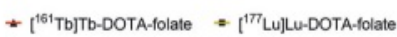

D

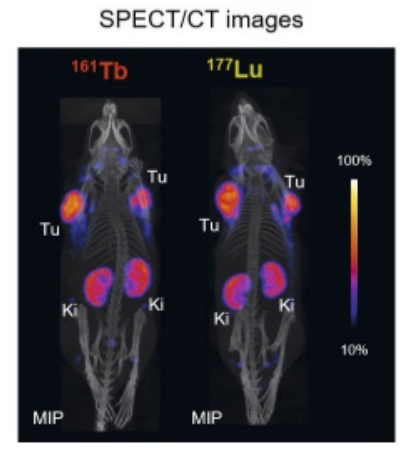


with $\left[{ }^{161} \mathrm{~Tb}\right] \mathrm{Tb}$-DOTA-folate than when using $\left[{ }^{177} \mathrm{Lu}\right] \mathrm{Lu}-\mathrm{DOTA}$ folate, which resulted in a significantly increased survival of these mice as compared to the group of mice treated with $\left[{ }^{177} \mathrm{Lu}\right] \mathrm{Lu}-$ DOTA-folate (Fig. 5C). ${ }^{[19]}$ The possibility of utilizing the emitted $\gamma$-radiation of terbium-161 for SPECT imaging was also successfully demonstrated in a tumor-bearing mouse that received $\left[{ }^{161} \mathrm{~Tb}\right] \mathrm{Tb}$-DOTA-folate (Fig. 5D). The KB tumor xenografts were visualized equally well in that mouse as was the case in a different mouse injected with $\left[{ }^{177} \mathrm{Lu}\right] \mathrm{Lu}$-DOTA-folate.

More recently, the superiority of terbium-161 over lutetium-177 was demonstrated in prostate cancer cells that express the prostate-specific membrane antigen (PSMA) using the clinically-employed PSMA-617 ligand. It was shown that $\left[{ }^{161} \mathrm{~Tb}\right] \mathrm{Tb}-$ PSMA-617 was significantly more effective in the killing of tumor cells in vitro compared to $\left[{ }^{177} \mathrm{Lu}\right] \mathrm{Lu}-\mathrm{PSMA}-617$ over a broad concentration range (Fig. 6A). ${ }^{[20]}$ These results received the Marie Curie Award in 2018 from the European Association of Nuclear Medicine (EANM) (Fig. 6B).

\subsection{Terbium-149 - Theragnostics at its Best}

Recent clinical data with targeted $\alpha$-radionuclide therapy (TAT) have revealed impressive results in mCRPC patients.[21] TAT's main advantage over $\beta^{-}$-particle therapy refers to the $\alpha$-particles' higher LET over a short path of only $50-100 \mu \mathrm{m}$ that results in a higher relative biological effectiveness (RBE). It is without doubt that the change in tumor burden before and after TAT is impressive and, probably of unprecedented value in the field of nuclear oncology. ${ }^{225}$ Ac-based TAT, however, raises questions about potential undesired side effects as a result of the complex decay chain of ${ }^{225} \mathrm{Ac}$, which results in several $\alpha$ - and $\beta^{-}$particle emissions from daughter nuclides. The so-called nuclear recoil effect causes release of the daughter radioactive nuclei from the targeting molecule and, thus, unwanted distribution of activity in the body, which may lead to radiotoxicity in healthy organs and tissue. ${ }^{[22]}$

Terbium-149 is an interesting $\alpha$-particle-emitting radiolanthanide thought to be a valid alternative to the currently-employedactinium-225 $\left(\mathrm{T}_{1 / 2}=9.9 \mathrm{~d}\right)$ and bismuth-213 $\left(\mathrm{T}_{1 / 2}=45 \mathrm{~min}\right)$, respectively. Its half-life of $4.1 \mathrm{~h}$ lies between bismuth-213 and actinium-225, which makes terbium-149 interesting for use in combination with fast-cleared small molecules that accumulate efficiently in the tumor tissue and are rapidly cleared from background organs. The decay chain of terbium-149 is considerably less complex than that of actinium-225, resulting in only one $\alpha$-particle emission (E $\alpha=$ $3.97 \mathrm{MeV} ; \mathrm{I}=17 \%)$. ${ }^{[23]}$ This may be advantageous with regard to the safety profile of ${ }^{149} \mathrm{~Tb}$-labeled radiopharmaceuticals.
In the literature, only few studies are reported that investigated terbium-149 for therapeutic purposes. Among those is an article of Beyer et al. who performed a preclinical experiment with ${ }^{149} \mathrm{~Tb}-$ rituximab in a leukemia mouse model.[24] The study demonstrated the feasibility to sterilize single cancer cells in circulation so that the mice survived over a period of 4 months, while untreated control mice developed tumor disease and reached the endpoint, which required euthanasia. ${ }^{[24]}$

At CRS, terbium-149 was initially investigated in a proofof-concept study using a DOTA-folate conjugate. ${ }^{[25]}$ A dosedependent inhibition of the KB tumor growth was observed in mice treated with [ $\left.{ }^{149} \mathrm{~Tb}\right] \mathrm{Tb}$-DOTA-folate, which led to a significantly increased median survival time as compared to untreated controls. ${ }^{[25]}$ More recently, we were able to conduct a preclinical study to investigate [ $\left.{ }^{149} \mathrm{~Tb}\right] \mathrm{Tb}-\mathrm{PSMA}-617$ with several groups of PC-3 PIP tumor-bearing mice (Fig. 7A). ${ }^{[26]}$ The resulting tumor growth curve was more favorable when the radioligand was injected twice ( $2 \times 3 \mathrm{MBq})$ on two consecutive days as compared to the effect of only one injection of the same total activity $(1 \mathrm{x}$ $6 \mathrm{MBq}$ ) (Fig. 7B). In both cases, the tumors did not, however, entirely disappear which indicated the need for more frequent injections. So far, it was not possible to further investigate and optimize the administration scheme of $\left[{ }^{149} \mathrm{~Tb}\right] \mathrm{Tb}-\mathrm{PSMA}-617$ to achieve tumor control over time, due to the limited availability of terbium-149 both in terms of frequency and quantity.

A unique feature of terbium-149 refers to the co-emission of $\beta^{+}$-particles (positrons) of an energy $\left(E \beta^{+}{ }_{\text {average }}=730 \mathrm{keV}, \mathrm{I}=\right.$ $7.1 \%$ ), suitable for PET imaging. The feasibility of PET imaging using terbium-149 was demonstrated for the first time using $\left[{ }^{149} \mathrm{~Tb}\right] \mathrm{Tb}-\mathrm{DOTANOC}$ in a mouse model of somatostatin-expressing xenografts. ${ }^{[23]}$ PET imaging was also successfully performed with $\left[{ }^{149} \mathrm{~Tb}\right] \mathrm{Tb}-\mathrm{PSMA}-617$ in tumor-bearing mice that enabled visualization of the PSMA-positive xenograft (PC-3 PIP tumor), but not the PSMA-negative xenograft (PC-3 flu tumor) located on the right and left shoulder of the mouse, respectively (Fig. 7C). ${ }^{[26]}$

Due to the $\beta^{+}$-particle co-emission, direct monitoring of the distribution of the radiopharmaceutical may be possible, which would make the application of terbium-149 considerably more interesting and safer than the use of currently employed $\alpha$-particleemitting radionuclides such as actinium-225. Dose estimations would be of unique value to better plan the following application and minimize the risk of damage to healthy tissues and organs. Although the preclinical data are scarce, terbium-149 is undoubtedly an interesting $\alpha$-particle emitter with great potential for clinical translation. Based on the experience gained from our research with terbium-161, it has become obvious that the chemical and
A

In vitro cell viability

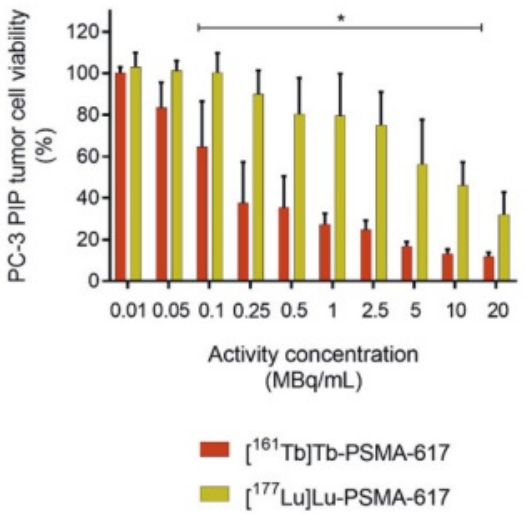

B

Marie Curie Award Medal

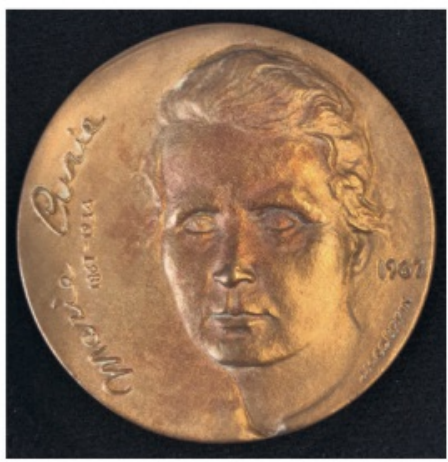

Fig. 6. (A) Results of in vitro cell viability studies performed with $\left.{ }^{161} \mathrm{~Tb}\right] \mathrm{Tb}-\mathrm{PSMA}-617$ and ${ }^{177} \mathrm{Lu}\left[{ }^{177} \mathrm{Lu}\right]-\mathrm{PSMA}-617$. The favorable effect of terbium- 161 over lutetium-177 was demonstrated by more effective reduction of cell viability when $\left[{ }^{161} \mathrm{~Tb}\right] \mathrm{Tb}-\mathrm{PSMA}-617$ was used. The bars represent the percentage of PC-3 PIP tumor cell viability after exposure to [ $\left.{ }^{161} \mathrm{~Tb}\right] \mathrm{Tb}-\mathrm{PSMA}-617$ and [177 $\left.\mathrm{Lu}\right]$ Lu-PSMA-617, respectively, compared to untreated control cells (set to $100 \%$ viability; average \pm SD); (B) Marie Curie Award medal received for the comparative study of [ $\left.{ }^{161} \mathrm{~Tb}\right] \mathrm{Tb}-\mathrm{PSMA}-617$ and [ [77 $\left.\mathrm{Lu}\right]$ Lu-PSMA-617. ${ }^{[20]}$ The data were adapted from Müller et al., Eur. J. Nucl. Med. Mol. Imaging 2019. 
A

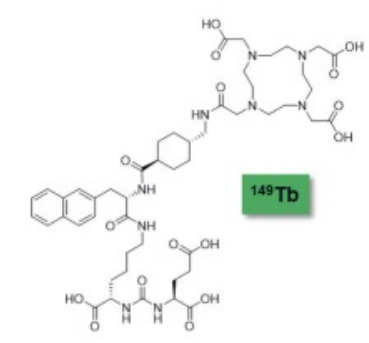

C

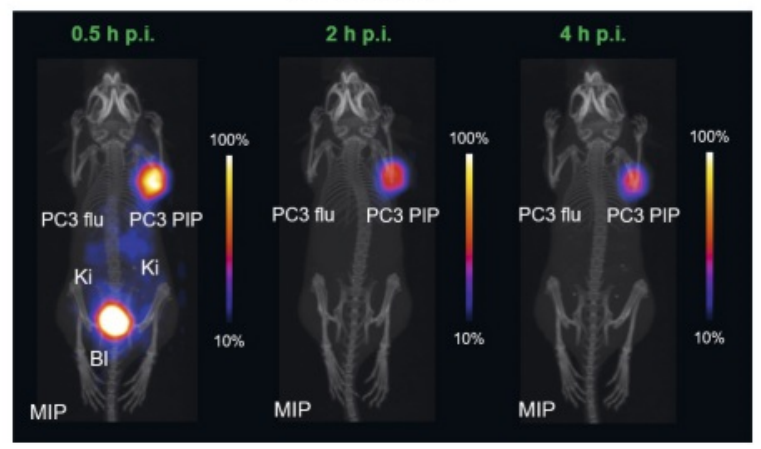

Fig. 7. (A) Chemical structure of PSMA-617. (B) Survival curve of mice treated with [149Tb]Tb-PSMA-617 applied in one (median survival: 26 days) or two injections (median survival: 36 days) as compared to untreated controls (median survival: 20 days); (C) PET/CT images shown as maximum intensity projections (MIPs) acquired $0.5 \mathrm{~h}, 2 \mathrm{~h}$ and $4 \mathrm{~h}$ post injection (p.i.) of [ $\left.{ }^{149} \mathrm{~Tb}\right] \mathrm{Tb}-\mathrm{PSMA}-617$ and $\left[{ }^{177} \mathrm{Lu}\right] \mathrm{Lu}$-DOTA-folate, respectively. The data were adapted from Umbricht et al., 2019, Sci. Reports. ${ }^{[2]]}$

pharmacokinetic behavior of Tb-labeled biomolecules is comparable to the ${ }^{177} \mathrm{Lu}$-labeled counterpart. ${ }^{[19,20]}$ This situation will facilitate the selection of suitable biomolecules to be used with terbium-149. The clinical translation of terbium-149 will critically depend on the availability of this interesting radionuclide, however, frequent production of terbium-149 in large quantities remains a major challenge. Several production routes have been proposed, [27] but the construction of dedicated production facilities, including mass separators that enable the isolation of terbium-149 from a mixture of produced terbium radioisotopes in the irradiated target, will be necessary. Currently, such endeavors are under discussion at several sites, hopefully enabling the production of terbium-149 in the near future and providing a new option for TAT in clinics.

\section{Conclusion and Further Considerations}

Radiopharmaceutical research is experiencing a stimulating and exciting period, hence, the expectations that more radiolabeled drugs will enter clinical trials in the near future are high. The strength of TRT is clearly the ability to address pathological conditions at an advanced stage when other therapeutic approaches are no longer an option. Different groups are currently also exploring the application of TRT in combination with other anticancer drugs including chemotherapeutics, ${ }^{[28]}$ tyrosine kinase inhibitors such as mTOR inhibitors, ${ }^{[29]}$ as well as immunotherapies ${ }^{[30]}$ to further improve the therapeutic outcome. ${ }^{[31]}$ The success of such approaches certainly depends on the targeting capacity and selectivity of a radiopharmaceutical, but also on the use of radionuclides with suitable decay properties tailored to individual indication.

What challenges will radiopharmaceutical research in Switzerland face in the future? Firstly, innovation with respect to new radionuclides requires expensive infrastructure, which needs regular upgrades and (partial) replacement. Such expensive endeavors have to be coordinated with the critical stakeholders, with the relevant parties being involved early in the decision and planning thereof. Secondly, a close dialogue with clinicians, in particular with oncologists and nuclear physicians, is essential in order to identify the clinical needs and relevant indications that should be addressed with TRT. Thirdly, radiopharmaceuticals are among the few drug types which can be developed from 'benchto-bedside' by academia in a reasonable time period, as they are applied in minute quantities and, thus, commonly well tolerated. This was shown for most of the currently-approved radiopharmaceuticals including $\left[{ }^{177} \mathrm{Lu}\right] \mathrm{Lu}-\mathrm{DOTATATE}$ and radiopharmaceuticals currently being tested in a Phase III clinical trial such as $\left[{ }^{177} \mathrm{Lu}\right] \mathrm{Lu}-\mathrm{PSMA}-617$. Meanwhile, conducting clinical trials has become difficult, however, and eventually prohibitively expensive for academic researchers, which were the main drivers for innovation until today. To overcome this challenge, a closer interaction and dialogue with the authorities will be necessary in order to find pragmatic solutions for the clinical testing of new radiopharmaceuticals, taking into account the low risk potential and the high benefit of this class of anticancer drugs.

\section{Acknowledgements}

Research on minigastrin has been supported by Swiss NAtional Science Foundation (SNSF) Sinergia grant (CRSII2_160805) and by the Technology Transfer Project of the Personalized Health and Related Technology (PHRT) initiative of the ETH Domain (PHRT-512). The research conducted with terbium-161 was supported by several funding institutions which are gratefully acknowledged: the Swiss National Science Foundation (SNSF: IZLIZ3_156800, 310030-156803); the Neuroendocrine Tumor Research Foundation (NETRF; Petersen Investigator Award 2018); the Personalized Health and Related Technology (PHRT-301), and the Swiss Cancer Research Foundation (KFS-4678-02-2019-R). With regard to the research conducted with terbium-149, the authors thank the ISOLDE RILIS team for efficient Dy ionization and the ISOLTRAP-MR-TOF-MS team for beam characterization. Support by ENSAR2 (EU H2020 project Nr. 654002) is gratefully acknowledged. Research on folates is supported by Merck \& Cie Schaffhausen and the research with PSMA targeting ligands by ITM Medical Isotopes $\mathrm{GmbH}$, Munich, Germany.

\section{Conflict of interest}

RS and MB hold patents on radiolabeled minigastrin analogues that have been licensed to Debiopharm. RS and CM are co-inventors of patents filed by Merck \& Cie, Schaffhausen, Switzerland and ITM Medical Isotopes $\mathrm{GmbH}$, Munich Germany, related to folate radioconjugates and PSMA radioligands, respectively.

Received: August 9, 2020

[1] C. Parker, S. Nilsson, D. Heinrich, S. I. Helle, J. M. O'Sullivan, S. D. Fossa, A. Chodacki, P. Wiechno, J. Logue, M. Seke, A. Widmark, D. C. Johannessen, P. Hoskin, D. Bottomley, N. D. James, A. Solberg, I. Syndikus, J. Kliment, S. Wedel, S. Boehmer, M. Dall'Oglio, L. Franzen, R. Coleman, N. J. Vogelzang, C. G. O'Bryan-Tear, K. Staudacher, J. Garcia-Vargas, M. Shan, O. S. Bruland, O. Sartor, A. Investigators, N. Engl. J. Med. 2013, 369, 213, https://doi.org/10.1056/NEJMoa1213755.

[2] J. Strosberg, G. El-Haddad, E. Wolin, A. Hendifar, J. Yao, B. Chasen, E. Mittra, P. L. Kunz, M. H. Kulke, H. Jacene, D. Bushnell, T. M. O'Dorisio, R. P. Baum, H. R. Kulkarni, M. Caplin, R. Lebtahi, T. Hobday, E. Delpassand, E. Van Cutsem, A. Benson, R. Srirajaskanthan, M. Pavel, J. Mora, J. Berlin, E. Grande, N. Reed, E. Seregni, K. Oberg, M. Lopera Sierra, P. Santoro, T. Thevenet, J. L. Erion, P. Ruszniewski, D. Kwekkeboom, E. Krenning, N.-T. Investigators, N. Engl. J. Med. 2017, 376, 125, https://doi.org/10.1056/NEJMoa1607427.

[3] C. Kratochwil, F. L. Giesel, M. Stefanova, M. Benesova, M. Bronzel, A Afshar-Oromieh, W. Mier, M. Eder, K. Kopka, U. Haberkorn, J. Nucl. Med. 2016, 57, 1170, https://doi.org/10.2967/jnumed.115.171397.

[4] R. Seifert, K. Kessel, K. Schlack, M. Weckesser, M. Bogemann, K. Rahbar, Eur. J. Nucl. Med. Mol. Imaging 2020, 47, 2106, https://doi.org/10.1007/s00259-020-04703-3.

[5] F. A. Verburg, B. Brans, F. M. Mottaghy, Methods 2011, 55, 230, https://doi.org/10.1016/j.ymeth.2011.06.002.

[6] J. C. Reubi, J. C. Schaer, B. Waser, Cancer Res. 1997, 57, 1377.

[7] M. Behe, W. Becker, M. Gotthardt, C. Angerstein, T. M. Behr, Eur. J. Nucl. Med. Mol. Imaging 2003, 30, 1140, https://doi.org/10.1007/s00259-003-1178-1. 
[8] M. Ocak, A. Helbok, C. Rangger, P. K. Peitl, B. A. Nock, G. Morelli, A Eek, J. K. Sosabowski, W. A. Breeman, J. C. Reubi, C. Decristoforo, Eur. J. Nucl. Med. Mol. Imaging 2011, 38, 1426, https://doi.org/10.1007/s00259011-1818-9.

[9] L. Aloj, C. Caraco, M. Panico, A. Zannetti, S. Del Vecchio, D. Tesauro, S. De Luca, C. Arra, C. Pedone, G. Morelli, M. Salvatore, J. Nucl. Med. 2004, 45, 485.

[10] C. Rottenburger, G. P. Nicolas, L. McDougall, F. Kaul, M. Cachovan, A. H. Vija, R. Schibli, S. Geistlich, A. Schumann, T. Rau, K. Glatz, M. Behe, E. R. Christ, D. Wild, J. Nucl. Med. 2020, 61, 520, https://doi.org/10.2967/jnumed.119.233031.

[11] W. A. Volkert, W. F. Goeckeler, G. J. Ehrhardt, A. R. Ketring, J. Nucl. Med. 1991, 32, 174

[12] LRC, https://www.psi.ch/de/lrc/radionuclide-development 2020, Paul Scherrer Institute.

[13] C. Müller, K. A. Domnanich, C. A. Umbricht, N. P. van der Meulen, Br. J. Radiol. 2018, 91, 20180074, https://doi.org/10.1259/bjr.20180074.

[14] C. Müller, K. Zhernosekov, U. Köster, K. Johnston, H. Dorrer, A. Hohn, N. T. van der Walt, A. Türler, R. Schibli, J. Nucl. Med. 2012, 53, 1951, https://doi.org/10.2967/jnumed.112.107540 jnumed.112.107540 [pii].

[15] M. T. Duran, F. Juget, Y. Nedjadi, F. Bochud, P. V. Grundler, N. Gracheva, C. Müller, Z. Talip, N. P. van der Meulen, C. Bailat, Appl. Radiat. Isot. 2020, 159, 109085, https://doi.org/10.1016/j.apradiso.2020.109085.

[16] S. Banerjee, M. R. Pillai, F. F. Knapp, Chem. Rev. 2015, 115, 2934, https://doi.org/10.1021/cr500171e.

[17] R. Valkema, S. A. Pauwels, L. K. Kvols, D. J. Kwekkeboom, F Jamar, M. de Jong, R. Barone, S. Walrand, P. P. Kooij, W. H. Bakker, J. Lasher, E. P. Krenning, J. Nucl. Med. 2005, 46 Suppl 1, 83S, https://doi.org/46/1_suppl/83S [pii].

[18] P. Bernhardt, E. Forssell-Aronsson, L. Jacobsson, G. Skarnemark, Acte Oncol. 2001, 40, 602, https://doi.org/10.1080/028418601750444141.

[19] C. Müller, J. Reber, S. Haller, H. Dorrer, P. Bernhardt, K. Zhernosekov, A. Türler, R. Schibli, Eur. J. Nucl. Med. Mol. Imaging 2014, 41, 476, https://doi.org/10.1007/s00259-013-2563-z.

[20] C. Müller, C. A. Umbricht, N. Gracheva, V. J. Tschan, G. Pellegrini, P. Bernhardt, J. R. Zeevaart, U. Köster, R. Schibli, N. P. van der Meulen, Eur. J. Nucl. Med. Mol. Imaging 2019, 46, 1919, https://doi.org/10.1007/s00259-019-04345-0.

[21] C. Kratochwil, F. Bruchertseifer, F. L. Giesel, C. Apostolidis, U. Haberhorn, A. Morgenstern, Eur. J. Nucl. Med. Mol. Imaging 2016, 43, S137.

[22] R. M. de Kruijff, H. T. Wolterbeek, A. G. Denkova, Pharmaceuticals 2015 , 8, 321, https://doi.org/10.3390/ph8020321.

[23] C. Müller, C. Vermeulen, U. Köster, K. Johnston, A. Türler, R. Schibli, N. van der Meulen, Eur. J. Nucl. Med. Mol. Imaging Radiopharm. Chem. 2017 1, 5, https://doi.org/10.1186/s41181-016-0008-2

[24] G. J. Beyer, M. Miederer, S. Vranjes-Duric, J. J. Comor, G. Kunzi, O. Hartley, R. Senekowitsch-Schmidtke, D. Soloviev, F. Buchegger, Eur. J. Nucl. Med. Mol. Imaging 2004, 31, 547, https://doi.org/10.1007/s00259003-1413-9.

[25] C. Müller, J. Reber, S. Haller, H. Dorrer, U. Köster, K. Johnston, K. Zhernosekov, A. Türler, R. Schibli, Pharmaceuticals 2014, 7, 353, https://doi.org/10.3390/ph7030353.

[26] C. A. Umbricht, U. Köster, P. Bernhardt, N. Gracheva, K. Johnston, R. Schibli, N. P. van der Meulen, C. Müller, Sci. Rep. 2019, 9, 17800, https://doi.org/10.1038/s41598-019-54150-w.

[27] G. J. Beyer, J. J. Comor, M. Dakovic, D. Soloviev, C. Tamburella, E. Hagebo, B. Allan, S. N. Dmitriev, N. G. Zaitseva, G. Y. Starodub, L. G. Molokanova, S. Vranjes, M. Miederer, I. Collaboration, Radiochim. Acta 2002, 90, 247, https://doi.org/10.1524/ract.2002.90.5_2002.247.

[28] a) P. G. Claringbold, P. A. Brayshaw, R. A. Price, J. H. Turner, Eur. J. Nucl. Med. Mol. Imaging 2011, 38, 302, https://doi.org/10.1007/s00259-0101631-x; b) S. Ballal, M. P. Yadav, N. A. Damle, R. K. Sahoo, C. Bal, Clin. Nucl. Med. 2017, 42, e457, https://doi.org/10.1097/RLU.0000000000001816.

[29] P. G. Claringbold, J. H. Turner, Cancer Biother. Radiopharm. 2015, 30, 261, https://doi.org/10.1089/cbr.2015.1876.

[30] a) R. Hernandez, K. L. Walker, J. J. Grudzinski, E. Aluicio-Sarduy, R. Patel, C. D. Zahm, A. N. Pinchuk, C. F. Massey, A. N. Bitton, R. J. Brown, P. M. Sondel, Z. S. Morris, J. W. Engle, C. M. Capitini, J. P. Weichert, Commun. Biol. 2019, 2, 79, https://doi.org/10.1038/s42003-019-03274; b) H. Chen, L. Zhao, K. Fu, Q. Lin, X. Wen, O. Jacobson, L. Sun, H Wu, X. Zhang, Z. Guo, Q. Lin, X. Chen, Theranostics 2019, 9, 7948 , https://doi.org/10.7150/thno.39203.

[31] G. Kong, R. J. Hicks, Curr. Treat. Options Oncol. 2019, 20, 77, https://doi.org/10.1007/s11864-019-0677-7.

\section{License and Terms}

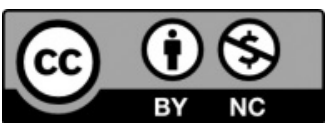

This is an Open Access article under the terms of the Creative Commons Attribution License CC BY NC 4.0. The material may not be used for commercial purposes.

The license is subject to the CHIMIA terms and conditions: (http:// chimia.ch/component/sppagebuilder/?view=page \&id=12).

The definitive version of this article is the electronic one that can be found at https://doi.org/10.2533/chimia.2020.939 